\title{
Working with 'difficult' clients from a systems perspective
}

\author{
Be Pannell \\ Australian College of Applied Psychology \\ Sydney, Australia
}

\begin{abstract}
Difficult or recalcitrant clients can be the bane of any executive coach's practice. Although cognitive behavioural approaches to coaching are effective for mainstream clients, they can fail to consider more nuanced aspects of the coaching dynamic. Psychodynamic theories, originating from Freud, seek to address unconscious patterns of interpersonal attachment and defensiveness that are considered the root cause of difficult interpersonal behaviour. However, these theories tend to focus on binary relationships and can fail to account for the overall system within which these relationships occur. In the following, a case conceptualisation framework for difficult clients is developed by combining psychodynamic models with family systems theory. Practical applications for coaching and coaching supervision are considered.
\end{abstract}

Keywords: difficult clients, psychodynamics, family systems, case conceptualisation

\section{Introduction}

Mainstream business psychology bookshelves are flooded with books on how to deal with difficult people, their approaches include ways to counterattack, defend or disarm the problem individual. Within coaching psychology, the dominant approach utilises cognitive behavioural strategies to address the cognitive distortions underlying the dysfunctional behaviour (Neenan \& Palmer, 2013), and if these strategies do not work, the client is often labelled uncoachable (Peterson, 2010), or pathological (Cavanagh, 2005). Although treatment of personality disorders and pathology is beyond the scope of the executive coach, less extreme personality styles such as narcissistic, aggressive, avoidant, antisocial and histrionic behaviour traits are common (Sperry, 2005).

Yet defining clients as 'difficult' is problematic as the evaluation tends to be based on a subjective assessment not grounded in empirical data, blaming a 
single person without considering the interpersonal and systemic dynamics that make up the difficult behaviour. Working with clients labelled difficult because of behavioural patterns such as arrogance, stubbornness, defensiveness, noncompliance and blame can challenge even the most experienced coach to move beyond the binary of us and them, to adopt a more nuanced understanding of the dynamics at play. In the following a case conceptualisation framework is developed to move beyond behavioural frameworks, and instead explore defensive and systemic patterns to identify alternative methods with which to intervene.

Case conceptualisation frameworks, originating from psychology, are maps that explore the connections between thoughts, feelings and behaviour. When developed in consultation with a coaching client, they can help establish a shared understanding of cognitive behavioural patterns and strategies for intervention. This paper extends the idea of cognitive case conceptualisation by mapping three psychodynamic theories of defensiveness within a family systems framework, to more thoroughly identify the factors contributing to difficult client behaviour. A case vignette is used to demonstrate how to apply this framework in coaching interventions.

\section{Psychodynamic Models}

Psychodynamic theory, originating with Freud's work on the unconscious, asserts that defensive behavioural patterns are responses to anxieties that arise from underlying conflicts within the psyche (Allcorn, 2006). Although much of Freud's work has been critiqued, particularly his ideas around sexuality, his theory of defences, extended by his daughter Anna, remains largely unchallenged. Freud's work on this theme has been developed in Horney's (1951) model of neurosis, which provides a solid theoretical and empirical basis for understanding the anxieties that drive difficult client behaviour. In addition, psychodynamic Attachment Theory (Bowlby, 2005) offers a nuanced framework to examine difficult interpersonal dynamics. As such, it is the combination of the three psychodynamic theories of defences, neurotic behaviour and attachment styles that forms the first part of this case conceptualisation.

According to Anna Freud, defence mechanisms such as denial, rationalisation, regression and projection are common reactions to underlying feelings of insecurity and anxiety, which are mostly unconscious. While some mechanisms such as denial of reality can be pathological, most allow an individual to maintain interpersonal relations at differing levels of functionality. 
Clients present as difficult in coaching when these coping mechanisms become maladaptive, which is particularly evident with regressive behaviours (Allcorn, 2006) such as tantrums, bullying, and shutdown.

According to Horney, maladaptive or neurotic tendencies stem from the overuse of three patterns of interpersonal coping. That is, clients who feel threatened will move away from others by withdrawing, move towards by becoming dependent, or against by turning aggressive. Clients who have a pattern of moving away will fear criticism and demonstrate independent, selfsufficient, critical and aloof behaviour, keeping their distance to maintain a sense of self-worth. Those who utilise a towards pattern will be dutiful and compliant, attempting to alleviate fears by becoming needed. Those who rely on the against tendency can use tyrannical, exploitative and aggressive behaviours to maintain control and protect themselves from harm (Horney, 1951). While the "against" pattern mentioned above is most often associated with difficult clients who display obvious signs of interpersonal aggression, all three patterns can be self-destructive in the workplace, and various 360 psychometric models (e.g., the Hogan Personality Inventory and The Leadership Circle) use Horney's tripartite classification in identifying these derailing tendencies.

According to attachment theory (Bowlby, 2005), interpersonal anxiety and maladaptive coping patterns develop from an insecure attachment between a preverbal child and its primary caregiver. Empirical evidence demonstrates that the four primary attachment styles developed in childhood will be replicated in adult interpersonal relationships and can be classified as secure, anxious, avoidant and disorganised. Securely attached infants develop a trusting relationship with others, and as adults are able to self-regulate their emotional response to unpredictable behaviour. Anxiously attached infants responded to emotionally unavailable caregivers by pursuing closeness at all costs, and as adults will be preoccupied with feelings of inadequacy. Avoidant infants learned to become self-reliant in response to unavailable caregivers and will experience discomfort with emotional cues from others and demands for closeness. Disorganised infants will tend to dissociate as adults and as such are beyond the scope of a coaching intervention.

\section{Psychodynamic Case Conceptualisation}

The fundamental principal of psychodynamic theories is that defensive behaviour is a maladaptive attempt to meet legitimate core needs (Drake, 2009). Conceptualising difficult client behaviour using a psychodynamic framework 
entails methodically mapping the defensive behaviours (e.g., aggression, rationalisation, projection), neurotic tendencies identified (away, towards, against) and the client's attachment style (secure, anxious, avoidant).

This mapping process is designed to support the client to step back and observe their patterns from a distance, rather than remain stuck in justifying their behaviour or blaming others. Becoming an impartial observer (taking the balcony view), can be aided by asking coaching questions such as: "what is being protected here?', 'what is the underlying coping pattern, or fear?' and 'how is this being compensated for?'. These questions support both the coach and client to begin to hypothesise as to the underlying core need (see Figure 1). Although it may take some time to build a level of trust for this to be explored fully with the client, the hypothesis helps the coach become less reactive to disruptive behaviour and can provide a core step in cultivating some empathy for the client's coping patterns (see Drake, 2009).

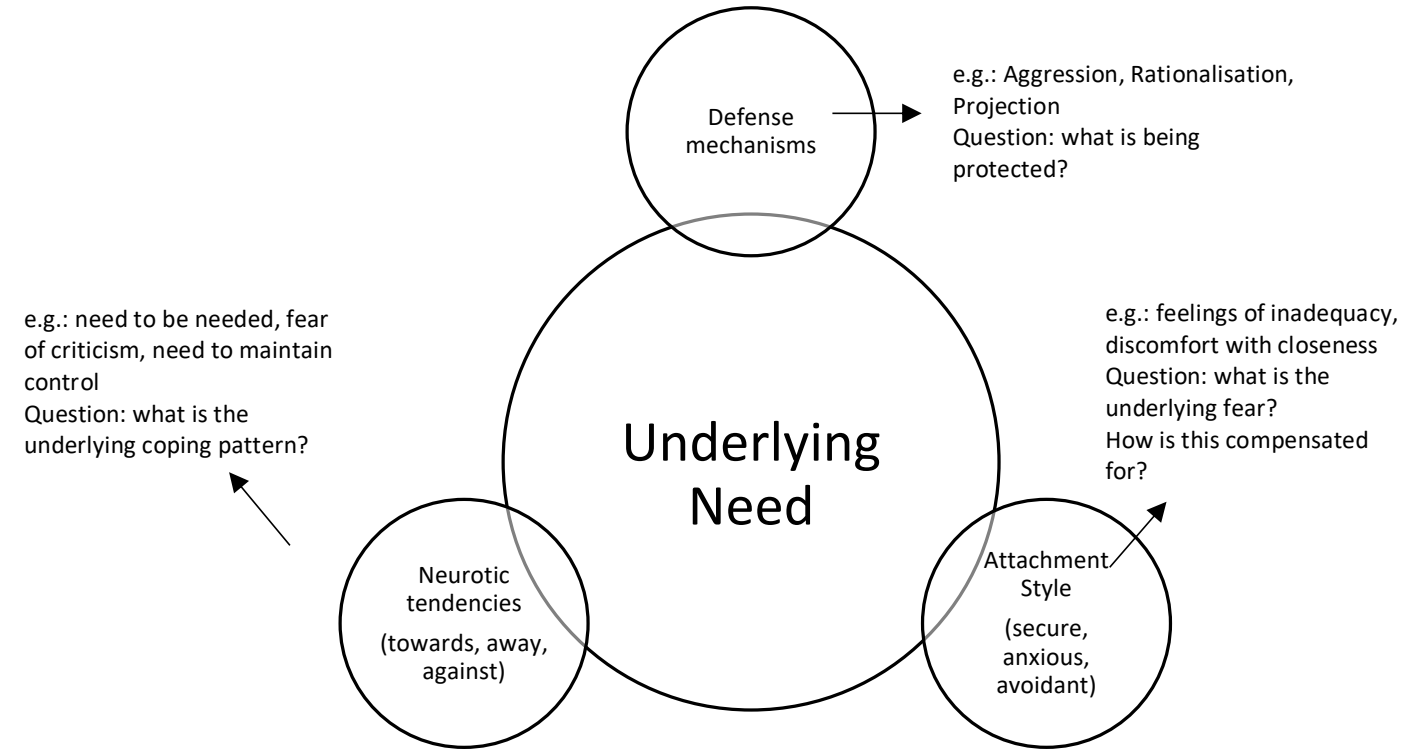

Figure 1: Psychodynamic Case Conceptualisation Framework

A psychodynamic case conceptualisation can include psychometric data, as well as coach and other 360 observations to reveal blind spots that the 'difficult' client may not bring to the coaching session. This case 
conceptualisation becomes a map in progress, whereby coach and client develop this together over a number of sessions. Additionally, being aware of the client's level of self-awareness, ambivalence and readiness to change are instrumental in developing the most effective coaching intervention.

Further, the psychodynamic framework can provide insight into the coach's own interpersonal patterns, particularly those that may get hooked into the client's in the process of counter transference. According to attachment theory, anxious and avoidant types tend to become enmeshed in a dysfunctional pattern of pursue and retreat, and it is only a securely attached individual that can down-regulate these patterns in another. Securely attached individuals have learned to self-regulate their emotions, independent of other people's behaviour, which frees up cognitive capacity for self-reflection and interpersonal attunement. Psychodynamically-informed supervision is recommended for coaches who feel particularly challenged by their difficult clients, to work through the unconscious patterns that the client may be triggering.

\section{Family Systems Theory}

Although psychodynamic frameworks are useful in identifying core needs that drive dysfunctional behaviour, they are largely based upon binary interpersonal relationships such as mother/child, employee/boss and client/coach. Systems theories recognise that these patterns exist within a much larger system that includes for example a network of other family members, work environments, processes and conceptual frameworks. Each element of a system interacts in seemingly unpredictable ways around attractors, moving towards homeostasis, and it is often only retrospectively that patterns emerge. Systems theories avoid attributing blame to a single individual or attractor, and instead map relationships and interactions in an attempt to identify alternative leverage points of intervention.

Many different systems theories have been proposed to explain the functioning of human systems (for a detailed overview see Mowles, Stacey \& Griffin, 2008). For the purpose of this paper, Family Systems Theory (Bowen, 1994) has been selected for use as it maps emotional interactions within a family unit, and is useful in understanding workplace dynamics (O'Neill, 2007). Bowen hypothesises that family members who are emotionally fused with each other will self-organise in ways to defuse the anxiety that arises from too much closeness or too large a distance within the family unit. A Bowenian intervention focuses on establishing awareness of these patterns, and 
encouraging each member to focus on themselves, and emotionally de-fuse from others (Brown, 1999).

Fundamental to Bowenian theory is the concept of 'triangling' (Brown, 1999), which is a stable dynamic between three fused family members to counter the tension between two of them (e.g., a child receiving excessive attention from parents who are not speaking to each other). Under long term stress, the pattern of triangling replicates and other triangles are formed that interlock and spread to include the wider community such as doctors, teachers and therapists (Brown, 1999). In the workplace, triangles develop when an individual has conflict with another person, but turns towards a third person for alliance and support, who in turn may form another triangle with others in the organisation and so on (O'Neill, 2007).

Using a Bowenian framework when working with so called difficult clients means recognising that difficult behaviour is part of a larger environment or system within which the behaviour occurs. No longer simply attributing difficult behaviour to a problem person, Bowenian systems thinking examines the dynamics between individuals, and also includes factors such as organisational culture, values, processes and power dynamics. These overlapping factors may form systems within systems, or triangles within triangles making intervention and change very difficult to achieve.

Just as in families, within organisations, a Bowenian systems approach can be utilised by a coach to addresses dysfunctional behaviour. This is achieved by firstly identifying patterns and triangling, and then supporting members of the triangle to move towards greater levels of functionality by developing the capacity to emotionally differentiate from each other, so as to reduce anxiety and subsequent reactivity.

\section{Bowen Family Systems Case Conceptualisation}

A Bowenian family systems case conceptualisation aims to slowly map the complex web of interpersonal relationships to provide a broader context for a client's difficult interpersonal behaviour. O'Connor and Cavanagh (2013) have used a similar framework to map positive and negative relationships within an organisation using social network analysis. In Bowen family systems, identifying where the client is emotionally fused with other people or environments (e.g., a work place culture) and mapping interlocking triangles helps to understand behavioural patterns (O'Neill, 2007) (See Figure 2). 
Fundamental to a Bowenian systemic case conceptualisation is mapping the interpersonal system established between the coach and the client. The transference and counter transference between coach and client includes power balances, unspoken contracts around emotional expression and unspoken boundary agreements that are likely to be replicated in each other's external relationships. Coaching supervision can be utilised to provide greater awareness and support so that the coach is able to maintain psychological differentiation within this dynamic.
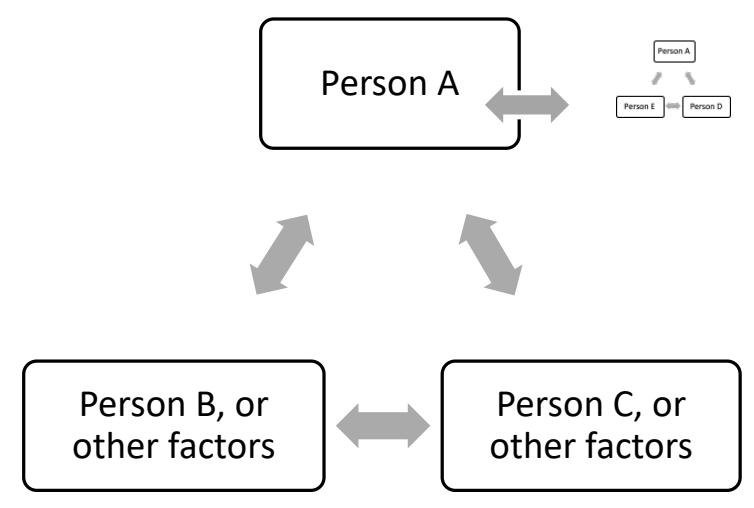

Figure 2: Mapping triangling in Bowen's Systems

\section{Psychodynamic, Bowenian Systems Conceptualisation}

Viewing psychodynamic patterns with a Bowenian systems lens entails avoiding attributing difficult behaviour within a client to any particular cause, but rather views the intra-personal patterns as part of a larger system containing other people, who also have their own internal psychodynamic system. Combining these psychodynamic patterns with a family systemic map of the client's interpersonal and organisational framework as a case conceptualisation, entails mapping many of these elements together. Once the psychodynamic system of defensive behaviours, neurotic tendencies and attachment styles are identified, the executive coach can work with the client to understand how these play out within different triangling frameworks (see Figure 3). 

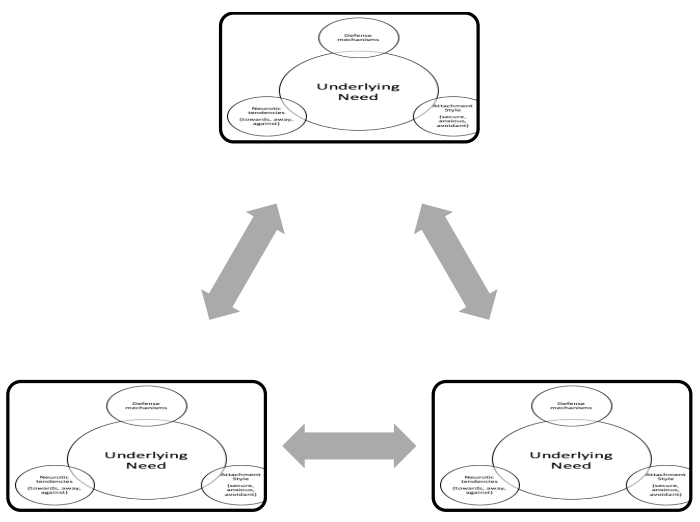

Figure 3: Psychodynamic, Bowenian Systems Conceptualisation

\section{Strategies for Intervention}

Fundamental to this approach is the ability of the coach to form a strong working alliance with the client so that difficult patterns may be raised in a nonthreatening, non-personal manner. This is aided by a dialogical framework that entails client and coach thinking together on the client's behaviours and developing a combined ownership of potential solutions (Bohm, 2013). As trust and respect are slowly developed, the coach can support the client to develop a metacognitive awareness of their attributional style by drawing attention to the habitual way the client interprets interpersonal interactions, including those between coach and client. By increasing metacognitive awareness, the client can develop the capacity to contribute to their case conceptualisation by identifying underlying patterns and drivers of their own behaviours.

\section{Case Vignette}

"Sam" was sent to coaching because of constant conflicts with her direct reports and boss. She was categorised as openly hostile, aggressive, critical and unpredictable. At the beginning of the coaching engagement she complained that it was a waste of time, and that she was not the problem. She was openly critical of the coaching process, and resisted attempts to encourage her to reflect on other people's perceptions of her behaviour. The first few coaching sessions were focused on allowing Sam to list all of her complaints about her direct reports and boss. 360 psychometric data showed Sam had strong tendencies to move away from others when threatened and adopt a critical stance towards 
them, except when she had had enough and would explode with rage. Early coaching sessions included systematically mapping the various interpersonal dynamics using the three psychodynamic theories, to develop a model which included her own habitual ways of responding to others (defensive patterns, moving away and avoiding, as shown in Figure 4).

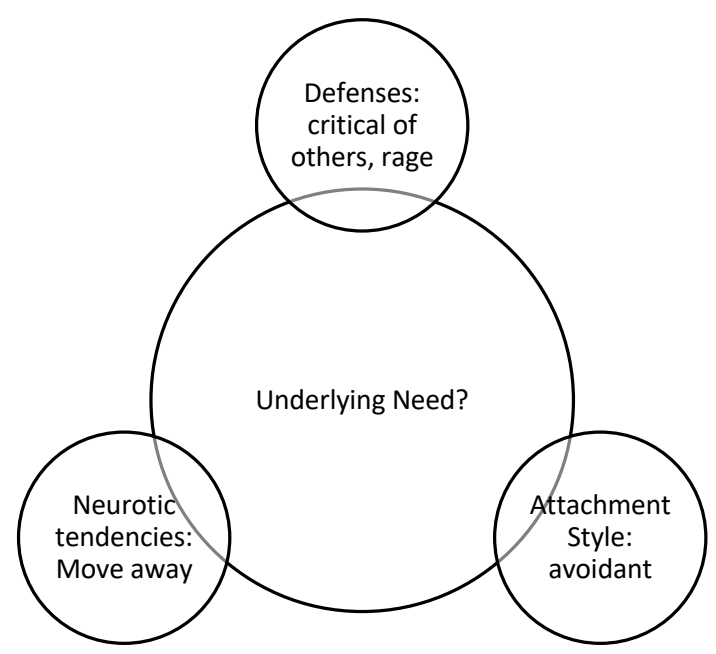

Figure 4: Psychodynamic Case Conceptualisation of Sam

As the case conceptualisation map developed in each session, Sam experienced being fully heard for perhaps the first time, and she was increasingly able to see her behaviour from a balcony view and talk through the assumptions and fears that underlay them. By applying Bowen's family systems theory to identify and explore the triangles between her direct reports and boss, she could identify how she would often leverage one against the other during conflict, and replicate triangles in other workplace relationships.

Within these coaching sessions, conversations turned to family dynamics and Sam began to understand the pattern of becoming aggressive as a dysfunctional attempt to be fully heard within a family system of poor communication. Once the underlying need to be heard was identified, coaching interventions focused on supporting Sam to emotionally de-fuse from others and to engage in difficult conversations while remaining calm and connected to the other person, which she first practiced within the coaching session. The more Sam was able to increase her communication skills, the less frustrated she 
felt, and the less she needed to rely on old attacking strategies to get her needs met (Figure 5).

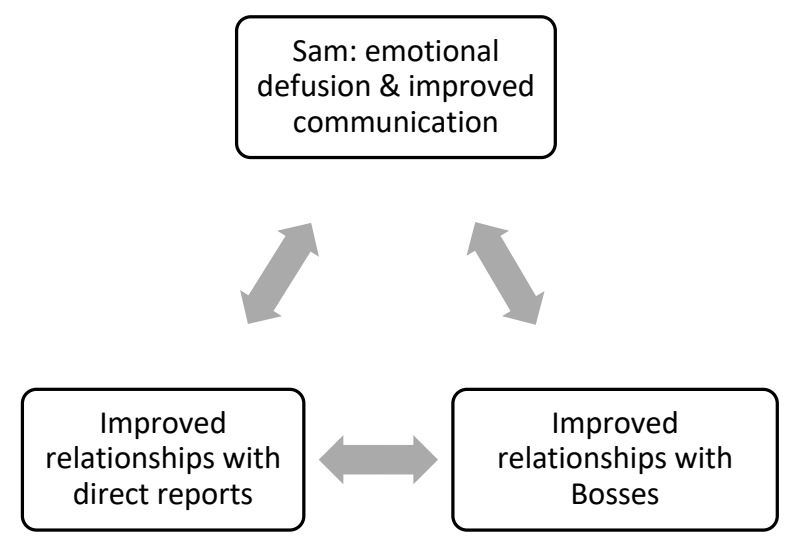

Figure 5: Results of addressing underlying need

\section{Directions for Future Research}

While there is much evidence of the effectiveness of psychodynamic interventions within coaching (Allcorn, 2006; Peltier, 2011) and some discussion of the application of Bowenian family systems theory (O'Neill, 2007), there is no known research that empirically investigates a combination of the two. Further research is needed to validate the different psychodynamic elements of this framework on a larger sample size, and to validate the assumption that difficult behaviour is a maladaptive pattern to meet deeper underlying needs. Additionally, the application of Bowen's Theory of triangling to organisational dynamics has not yet been empirically validated, nor the impact of coaching on de-fusing these triangles. Future research might also use the Social Network Analysis methodology (O’Connor \& Cavanagh, 2013) as an alternative to Bowen's triangling systems to understand the systemic elements of difficult client behavioural patterns.

\section{Conclusion}

Working with clients who demonstrate difficult behaviour demands a high level of interpersonal skill within the coach, including the ability to form a working alliance with a potentially hostile client that includes providing challenging feedback on behavioural and interpersonal patterns. Although many coaching techniques exist to address cognitive and behavioural issues, few 
consider how a 'difficult' client is conceptualised or the systemic factors impacting their behaviour. This paper outlined a unique perspective on conceptualising difficult client behaviour by combining three different psychodynamic theories with Bowen family systems theory to re-conceptualise difficult behaviour as a maladaptive attempt to meet deeper underlying needs. By mapping interlocking interpersonal triangles against defence mechanisms, neurotic strategies and attachment styles, this paper argues that the executive coach is better equipped to identify strategies for building trust and leverage points for intervention. As coaching interventions continue to focus on novel ways of theorising and addressing the core drivers of difficult client behaviour, coaching will continue to advance beyond cognitive behavioural formulations. Once underlying patterns are understood and explored, the client can be supported to become less defended and hostile, with less outward focus on other people's behaviour. This in turn can create greater psychological space for healthy attachment with the coach and greater capacity for metacognition and ultimately behavioural change.

\section{References}

Allcorn, S. (2006). Psychoanalytically Informed Executive Coaching. In D. R. Stober \& A. M. Grant (Eds.), Evidence Based Coaching Handbook: Putting Best Practices to Work for Your Clients. Wiley (pp. 129-152).

Brown, J. (1999). Bowen Family Systems Theory and Practice: Illustration and Critique. Australian and New Zealand Journal of Family Therapy, 20(2), 94-103

Bohm, D. (2013). On Dialogue. Routledge.

Bowen, M. (1994). Family Therapy in Clinical Practice. Rowman \& Littleford Publishers

Bowlby, J. (2005). A Secure Base. Routledge.

Cavanagh, M. (2005). Mental-Health Issues and Challenging Clients in Executive Coaching. In M. Cavanagh, A. M. Grant, \& T. Kemp (Eds.), Evidence-based Coaching: Theory, research and practice from the behavioural sciences (pp. 21-36). Australian Academic Press.

Drake, D. B. (2009). Using attachment theory in coaching leaders: The search for a coherent narrative. International Coaching Psychology Review, 4(1), 49-58.

Horney, K. (1951). Neurosis and Human Growth: The struggle toward selfrealization. Routledge.

Mowles, C., Stacey, R., \& Griffin, D. (2008). What contribution can insights from the complexity sciences make to the theory and practice of 
development management? Journal of International Development: The Journal of the Development Studies Association, 20(6), 804-820.

Neenan, M., \& Palmer, S. (2013). Cognitive behavioural coaching in practice: An evidence-based approach. Routledge.

O'Connor, S., \& Cavanagh, M. (2013). The coaching ripple effect: The effects of developmental coaching on wellbeing across organisational networks. Psychology of Well-Being: Theory, Research and Practice, $3(2)$.

O'Neill, M. B. (2007). Executive Coaching with Backbone and Heart: A Systems Approach to Engaging Leaders with Their Challenges (2 ${ }^{\text {nd }}$ Edition). Wiley.

Peltier, B. (2011). The Psychology of Executive Coaching: Theory and Application. Taylor \& Francis.

Peterson, D. (2010). Good to Great Coaching: Accelerating the Journey. In G. Hernez-Broome \& L. A. Boyce (Eds.), Advancing Executive Coaching: Setting the Course for Successful Leadership Coaching. Wiley (pp. 83102).

Sperry, L. (2005). Handbook of Diagnosis and Treatment of DSM-IV Personality Disorders ( $2^{\text {nd }}$ Edition.). Taylor \& Francis.

\author{
Author Contact \\ Dr Be Pannell \\ Associate Professor \\ Australian College of Applied Psychology \\ 255 Elizabeth Street \\ Sydney, NSW \\ Australia \\ E: be.pannell@acap.edu.au
}

